\title{
Genetic Inferences and Breeding Implications from Analysis of Cranberry Germplasm Anthocyanin Profiles
}

\author{
Nicholi Vorsa ${ }^{1}$ and James Polashock ${ }^{2}$ \\ Philip E. Marucci Center for Blueberry and Cranberry Research and Extension, Rutgers University, \\ Chatsworth, NJ 08019 \\ David Cunningham ${ }^{3}$ and Robin Roderick ${ }^{4}$ \\ Ocean Spray Cranberries, Inc., One Ocean Spray Drive, Lakeville-Middleboro, MA 02349
}

\begin{abstract}
AdDITIONAL INDEX WORDS. Vaccinium macrocarpon, fruit, methoxylation, glycosylation, cyanidin, peonidin
Abstract. Adiversity of anthocyanins exists among angiosperm species. Studies indicate that various anthocyanins differ in antioxidant potential, their bioavailability, and stability during processing. The fruit of the American cranberry, Vaccinium macrocarpon Ait., is recognized as having six anthocyanins, composed largely of 3-O-galactosides and 3-O-arabinosides, and to lesser amount $(\approx 6 \%), 3-O$-glucosides of the aglycones cyanidin and peonidin. This study analyzed proportions of these six anthocyanins from $>\mathbf{2 5 0}$ accessions of a germplasm collection over harvest dates. Fruit samples from 78 selected accessions, based on the first year analysis, were also analyzed a second year. Principal component analysis identified general negative relationships between the proportions of cyanidin versus peonidin, arabinosides versus glucosides, and galactosides versus arabinosides and glucosides. These relationships were consistent across the 2 years. Most variation in germplasm anthocyanin profiles reflected variation of cyanidin versus peonidin proportions, with cyanidin to peonidin ratios ranging from 3.6:1 to 0.5:1. Variation for glycosylation profiles was also evident, with galactoside proportions ranging from $64 \%$ to $75 \%$, arabinoside proportions ranging from $20 \%$ to $33 \%$, and glucoside proportions ranging from $3 \%$ to 9\%. Evidence for both significant qualitative and quantitative genetic variation exists for the methoxylation of cyanidin to peonidin. Significant quantitative genetic variation is also apparent for glycosylation.
\end{abstract}

Significant differences exist among anthocyanins as to their antioxidant potential (Cao et al., 1996). Antioxidant potential appears to be a function of both the glycoside, as well as the specific aglycone, i.e. cyanidin, malvidin, pelargonin, etc. (Satue-Gracia et al., 1997; Wang et al., 1997). For example, Wang et al. (1997) found cyanidin-3-glucoside to have $\approx 75 \%$ greater antioxidant activity than cyanidin-3-galactoside. Cyanidin-3-glucoside was found to have $>90 \%$ greater antioxidant activity than peonidin3 -glucoside (Wang et al., 1997). Evidence is also increasing for the significant role that the carbohydrate moiety plays in the absorption of numerous flavonoids in the gastro-intestinal tract (Gee et al., 2000; Hollman and Katan, 1997; Hollman et al., 1997; Setchell et al., 2001). Glucoside conjugates appear to be the most bioavailable. Hollman and Katan (1997) found that quercetin3 -glucoside was absorbed to a significantly greater degree than quercetin-3-rutinoside in human subjects. Mizuma et al. (1994) in studies with rat intestines found flavonols as glucosides were absorbed to a greater degree than the galactosides. Anthocyanins also differ in their stability during processing (Wrolstad et al., 1994).

The American cranberry (Vaccinium macrocarpon Ait.) is recognized for its brilliant red fruit, which is a result of a fairly high abundance of anthocyanins in the fruit epidermal tissues. The anthocyanins of $\mathrm{V}$. macrocarpon are mainly the 3-O-glycosides of cyanidin and peonidin. Galactosides and arabinosides of cyanidin and peonidin have been reported to be the most abundant, with lesser ( $\approx 6 \%)$ amounts of glucoside anthocyanins (Hong and

Received for publication 19 Oct. 2002. Accepted for publication 18 Feb. 2003. We thank Kate Brilliant and Sarah Vannozzi for their technical assistance, and Joseph DeVerna, for helpful suggestions and critically reviewing the manuscript.

'Professor; corresponding author.

${ }^{2}$ Assistant research director. Current address: USDA-ARS-Fruit Lab, Chatsworth, NJ 08019.

3Principal scientist.

${ }^{4}$ Senior scientist.
Wrolstad, 1990). Although total anthocyanin content (TAcy) of fruit is one of the major traits evaluated in cranberry breeding (Sapers et al., 1983a), information on variation of proportions of individual anthocyanins is generally lacking. Earlier studies concluded that there were either no or very little qualitative or quantitative differences in the proportions of individual anthocyanins among the cranberry varieties or harvest dates studied (Sapers et al.,1983b; Sapers and Hargrave, 1987). These studies did not consider the proportions of glucosides, most likely assuming they were of little consequence. The proportion of cyanidin to peonidin anthocyanins was determined to be about equal (Hong and Wrolstad, 1986, 1990; Sapers and Hargrave, 1987), however, these studies evaluated only a limited number of varieties.

We have assembled a large collection of cranberry germplasm accessions from cultivated beds. Analyzing anthocyanin profiles of a broad array of germplasm over harvest dates and years may provide insight into the genetic variation and inter-relationships among the specific anthocyanins in cranberry, as well as providing useful information for breeding of desirable anthocyanin profiles. The objectives of this study were to 1) analyze the proportions of the individual anthocyanins in a broad array of germplasm obtained from cultivated beds over harvest dates and years to better assess the variation for anthocyanin profiles in American cranberry, 2) identify relationships, or possible tradeoffs, among the anthocyanins using principal component analysis, and 3) identify varieties with non-typical anthocyanin profiles.

\section{Materials and Methods}

Plant material. Germplasm used in this evaluation consisted of 252 clonal accessions collected between 1988 and 1993 from cultivated beds in Massachusetts, New Jersey, Oregon, Washington, and Wisconsin, which were established in $2.3-\mathrm{m}^{2}$ field plots. Germplasm plots were located in one of two 0.25 -ha beds (Beds 1 and 4) at the Rutgers (N.J.) Univ. P.E. Marucci Center. 
Bed 1 has a 25-cm-deep washed sand base, while Bed 4 consists of berryland soil.

Fruit SAMPles, TACY AND HPLC anAlysis. Fruit samples from 252 plots were harvested from $930-\mathrm{cm}^{2}$ areas (minimally $30 \mathrm{~cm}$ from plot edge) from each plot on 5 Sept. and 5 Oct. 1998. In 1999, 78 accessions were selected (accessions were selected to represent the full range of variation based on 1998 principal component analysis) for fruit samples on 20 Sept. and 11 Oct. The data obtained for each fruit sample harvested were total weight, berry count, mean fruit weight, damaged fruit weight and number and chemical analyses are described below.

For both years, fruit samples $(50 \mathrm{~g})$ were frozen at $-20{ }^{\circ} \mathrm{C}$. The samples were analyzed randomly across plots (accessions) and harvest dates. The $50 \mathrm{~g}$ frozen fruit sample along with $60 \mathrm{~mL}$ of hot $\left(50^{\circ} \mathrm{C}\right)$ tap water were placed in a blender and macerated for 3 min. From the resultant slurry, the total anthocyanins (TAcy) were determined using a standard spectrophotometric assay adapted from Sapers et al. (1983b). Concurrently, the sample was also prepared for analysis of total phenolics, individual anthocyanins and total anthocyanins by HPLC (TAcy-HPLC). The slurry was centrifuged $\left(40,790 \times g, 10 \mathrm{~min}, 25^{\circ} \mathrm{C}\right)$, the supernatant filtered $(0.45 \mu \mathrm{m})$, the filtrate sealed in a $2 \mathrm{~mL}$ amber glass injection vial, and frozen until analysis. The HPLC method used was adapted from LamuelaRaventos and Waterhouse (1994), substituting catechin for gallic acid as a standard. The HPLC was a binary pump system (Hewlett Packard 1100) with a variable diode-array detector. UV-visible spectra were recorded at 280,320, and $520 \mathrm{~nm}$. The column was a Prodigy ODS-3, $5 \mu \mathrm{m}, 250 \times 4.6 \mathrm{~mm}$, protected with a Prodigy guard ODS-3, $5 \mu \mathrm{m}, 30 \times 4.6 \mathrm{~mm}$. Separations were carried out using the following conditions: $1 \mathrm{~mL} \cdot \mathrm{min}^{-1}$ flow rate, $25-\mu \mathrm{L}$ injection volume; column compartment temperature $30^{\circ} \mathrm{C}$; solvent A, $2 \%(\mathrm{v} / \mathrm{v})$ acetic acid in water; solvent $\mathrm{B}, 80 \%(\mathrm{v} / \mathrm{v})$ acetonitrile and $20 \%$ solvent $\mathrm{A}$; elution with linear gradients from $0 \% \mathrm{~B}$ (balance $100 \% \mathrm{~A}$ ) for $3 \mathrm{~min}$, from $0 \% \mathrm{~B}$ to $4 \% \mathrm{~B}$ in $3 \mathrm{~min}$, from $4 \% \mathrm{~B}$ to $10 \% \mathrm{~B}$ in $9 \mathrm{~min}$, from $10 \% \mathrm{~B}$ to $15 \% \mathrm{~B}$ in $15 \mathrm{~min}$, from $15 \% \mathrm{~B}$ to $23 \% \mathrm{~B}$ in $20 \mathrm{~min}$, from $23 \% \mathrm{~B}$ to $25 \%$ in $10 \mathrm{~min}$, from $25 \% \mathrm{~B}$ to $30 \% \mathrm{~B}$ in $6 \mathrm{~min}$, from $30 \% \mathrm{~B}$ to $50 \% \mathrm{~B}$ in $14 \mathrm{~min}$, from $50 \% \mathrm{~B}$ to $80 \% \mathrm{~B}$ in $3 \mathrm{~min}$, and then back to $0 \% \mathrm{~B}$ in $2 \mathrm{~min}$, followed by a 20 min post time. Individual anthocyanins were quantified using a standard of cyanidin-3-galactoside at $520 \mathrm{~nm}$. TAcy-HPLC was calculated as the sum of areas representing the peaks at retention time (RT) for the six anthocyanins.

STAtistical anAlysis. Statistical analysis employed SAS 8.0 for Microsoft Windows, SAS Institute, Inc., Cary, N.C. Procedures used were PROC CORR for Pearson's correlation coefficients, PROC PRIN for principal component analysis, and PROC GLM for analysis of variance with Type III sums-of-squares for testing bed, harvest date and variety effects.

Table 1. Eigenvalue percentages for principal components (PC) 1-5 vectors derived from the absolute levels of six cranberry anthocyanins in the germplasm collection.

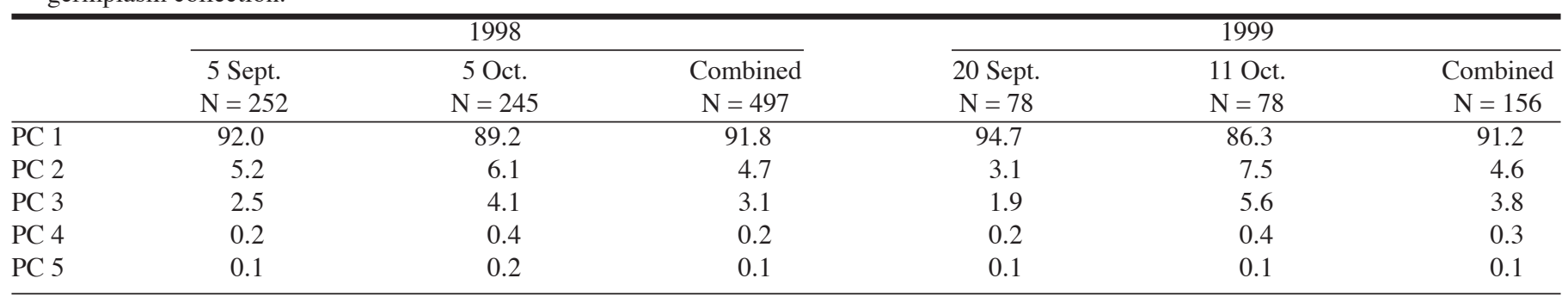

Table 2. Pearson's correlation coefficients for correlation among levels of the six anthocyanins (cyanidin-3-arabinoside, cyanidin-3-galactoside, cyanidin-3-glucoside, peonidin-3-arabinoside, peonidin-3-galactoside, peonidin-3-glucoside), with one another as absolute values (above diagonal) and as percentages of the total anthocyanins of the sample (below diagonal).

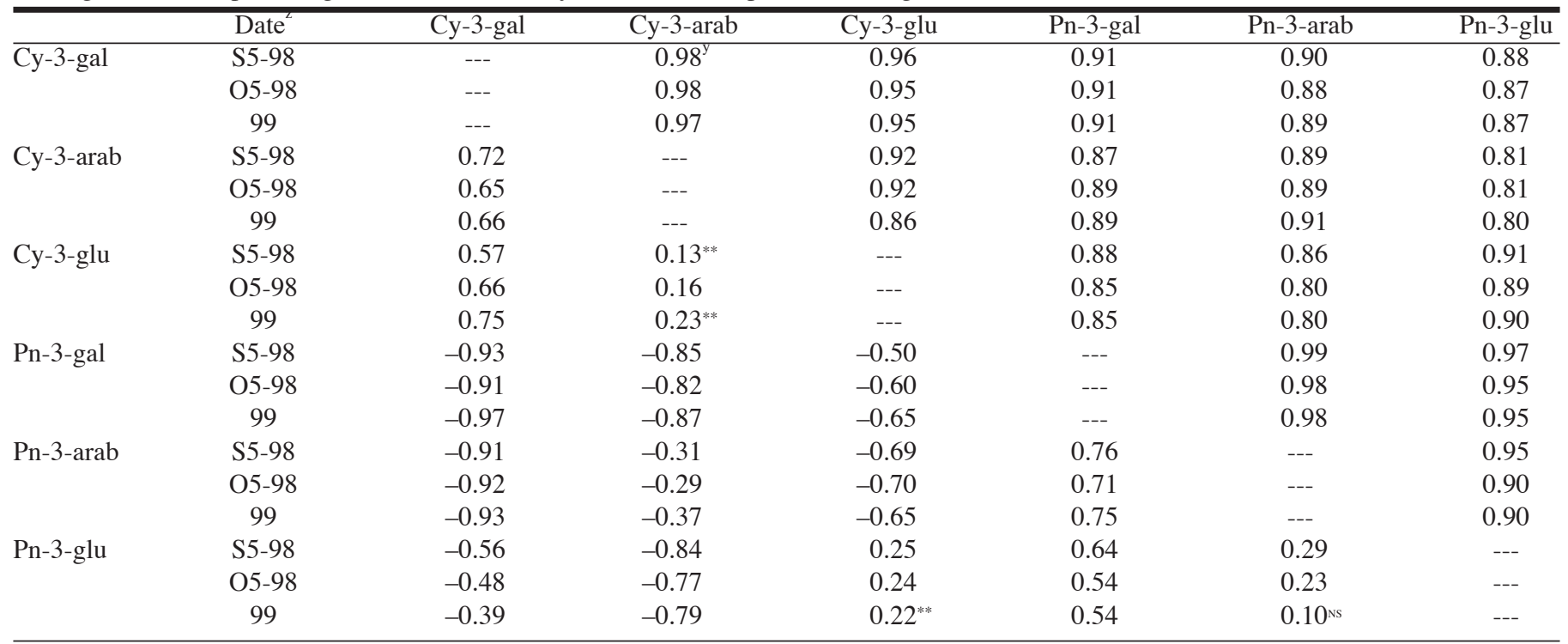

${ }^{\mathrm{z}}$ Harvest dates are S5-98 (5 Sept. 1998), O5-98 (5 Oct. 1998) and 99 (20 Sept. and 11 Oct. 1999).

yAll values significant at less than $P=0.0001$, unless noted otherwise.

ss,***Nonsignificant or significant at $P=0.01$, respectively. 


\section{Results}

CRANBERRY ANTHOCYANIN PROFILE VARIATION: INTER-RELATIONSHIPS OF INDIVIDUAL ANTHOCYANINS. TAcy-HPLC quantities (the six anthocyanins combined) ranged from $9.6 \mu \mathrm{g} \cdot \mathrm{g}^{-1}$ (US88-85, 5 Sept.) to $1,500 \mu \mathrm{g} \cdot \mathrm{g}^{-1}$ (US88-119, 5 Oct.) in 1998. In 1999, total anthocyanins ranged from $31.1 \mu \mathrm{g}^{-\mathrm{g}^{-1}}$ (US 88-92, 20 Sept.) to $1,108.5 \mu \mathrm{g} \cdot \mathrm{g}^{-1}$ (US88-119, 11 Oct.). Total anthocyanins (TAcy) ranged from 3 to $97 \mathrm{mg} / 100 \mathrm{~g}$ fruit in 1998 and from $<1$ to 125 $\mathrm{mg} / 100 \mathrm{~g}$ fruit in 1999. TAcy was fairly highly correlated with TAcy-HPLC values, with $r=0.88(P<0.001, \mathrm{~N}=497)$ in 1998 and $r=0.95(P<0.001, \mathrm{~N}=164)$ in 1999 .

Principal component analysis indicates the majority of the variation was due to differences in overall absolute values of the six anthocyanins, resulting from differences between varieties and harvest dates. The first principal component (PC1) accounts for between $86.3 \%$ to $94.7 \%$ of the variation (Table 1), with about equal weightings for the absolute levels of the six anthocyanins for both 1998 and 1999 (data not given). Variation arising from differences in relative proportions of the six anthocyanins accounted for about $8 \%$ of the total variation (Table 1). Within harvest dates, the variation attributable to differences in proportions of the six anthocyanins ranged from 5.3\% (20 Sept. 1999) to $13.7 \%$ (11 Oct. 1999) (Table 1). The absolute values of the individual anthocyanins were all significantly positively correlated with each other (Table 2, above diagonal). The highest correlation was observed between the galactosides and arabinosides of cyanidin $(r=0.98)$ and peonidin $(r=0.98)$, respectively. The lowest correlations were cyanidin-3-arabinoside with peonidin-3-glucoside $(r=$ $0.81)$ and cyanidin-3-glucoside with peonidin-3-arabinoside $(r=$ 0.86) (Table 2, above diagonal).

To identify relationships among the levels of six anthocyanins, the levels of the six anthocyanins were analyzed as proportions of the total anthocyanins within a sample. Relationships among the proportions of the six anthocyanins are evident in the first (PC1), second (PC2) and third (PC3) principal components, and were essentially consistent across harvest dates (not shown) and the two years (Table 3 ). PC1 accounted for $\approx 66 \%$ of the total variation in the six anthocyanin proportions.
The weightings in PC1 indicate that there is a general negative correlation between cyanidin versus peonidin glycoside proportions for fruit harvested both years, although the signs were reversed between the years (Table 4). The assignment of sign (positive or negative) to variables in PROC PRIN follows the arbitrary rule that the sum of eigenvectors is greater than zero (Anonymous, SAS Institute, Inc.). Except for the low (but significant) positive correlation between cyanidin-3-glucoside and peonidin-3-glucoside, the relationships between the cyanidin versus peonidin glycosides were all negative, varying in magnitude from $r=-0.29$ between arabinosides, to $r=-0.93$ between the galactosides (Table 2, below diagonal). PC2 accounted for $27.2 \%$ to $32.2 \%$ of the total variation in anthocyanin proportions (Table 3 ). The PC2 eigenvectors suggest a general negative relationship between arabinosides and glucosides. The glucosides of cyanidin and peonidin have positive weightings, while the arabinosides of cyanidin and peonidin are negatively weighted, indicating a negative correlation between the glucosides and arabinosides (Table 4). The galactoside anthocyanins in PC2 were essentially neutral, as indicated with weightings nearer to zero ranging from 0.03 to 0.12 . PC3 accounted for $3 \%$ to $5 \%$ of the variation (Table 3 ). The weightings of the PC3 eigenvectors suggest a general negative relationship between the galactoside proportions versus proportions of both arabinosides and glucosides, and were consistent between the 2 years (Table 4). The galactosides of cyanidin and peonidin had about equal negative weightings, while the weightings for glucosides and arabinosides of both cyanidin and peonidin were positive, indicating galactosides are negatively associated with that of arabinosides and glucosides. It appears that although the glucoside and arabinoside proportions are still positively correlated with the galactoside proportions within an aglycone class, the correlation values are only moderate, ranging from $r=0.54$ to 0.76 (Table 4, below diagonal). The weightings of the arabinosides appeared to be slightly higher than for the glucosides.

Anthocyanin profile variation. A plot of PC1 versus PC2 displays the variation for cyanidin versus peonidin anthocyanin proportions (PC1), and arabinose versus glucose anthocyanin proportions (PC2), respectively, in 1998 (Fig. 1). The majority

Table 3. Eigenvalue proportions for principal components (PC) 1-4 vectors derived from the proportions of six cranberry anthocyanins in the germplasm collection.

\begin{tabular}{|c|c|c|c|c|c|c|}
\hline & \multicolumn{3}{|c|}{1998} & \multicolumn{3}{|c|}{1999} \\
\hline & $\begin{array}{c}5 \text { Sept. } \\
\mathrm{N}=252\end{array}$ & $\begin{array}{c}5 \text { Oct. } \\
\mathrm{N}=245\end{array}$ & $\begin{array}{c}\text { Combined } \\
\mathrm{N}=497\end{array}$ & $\begin{array}{c}20 \text { Sept. } \\
N=78\end{array}$ & $\begin{array}{l}11 \text { Oct. } \\
\mathrm{N}=78\end{array}$ & $\begin{array}{c}\text { Combined } \\
\mathrm{N}=156\end{array}$ \\
\hline$\overline{\mathrm{PC}} 1$ & 66.3 & 66.1 & 66.7 & 66.6 & 63.6 & 66.7 \\
\hline PC 3 & 3.9 & 4.7 & 4.2 & 4.8 & 3.3 & 4.5 \\
\hline PC 4 & 1.2 & 1.5 & $<1$ & 1.2 & 0.7 & 1.1 \\
\hline
\end{tabular}

Table 4. Eigenvectors for principal components 1-3 (PC1, PC2 and PC3) for proportions of six cranberry anthocyanins in cranberry germplasm across two harvest dates for 1998 and 1999.

\begin{tabular}{|c|c|c|c|c|c|c|}
\hline \multirow[b]{2}{*}{ Anthocyanin } & \multicolumn{2}{|c|}{ PC1 } & \multicolumn{2}{|c|}{$\mathrm{PC} 2$} & \multicolumn{2}{|c|}{ PC3 } \\
\hline & $1998^{z}$ & $1999 y$ & $1998^{z}$ & $1999 y$ & $1998^{z}$ & $1999 y$ \\
\hline Cy-3-gal & -0.49 & 0.49 & 0.12 & 0.10 & -0.26 & -0.28 \\
\hline Cy-3-glc & -0.26 & 0.35 & 0.64 & 0.50 & 0.42 & 0.40 \\
\hline Pn-3-gal & 0.49 & -0.49 & 0.03 & 0.09 & -0.31 & -0.28 \\
\hline Pn-3-glc & 0.32 & -0.24 & 0.57 & 0.65 & 0.27 & 0.37 \\
\hline Pn-3-arab & 0.43 & -0.43 & -0.32 & -0.33 & 0.59 & 0.56 \\
\hline
\end{tabular}

${ }^{\mathrm{z}}$ Harvest dates are 5 Sept. and 5 Oct.

yHarvest dates are 20 Sept. and 11 Oct. 


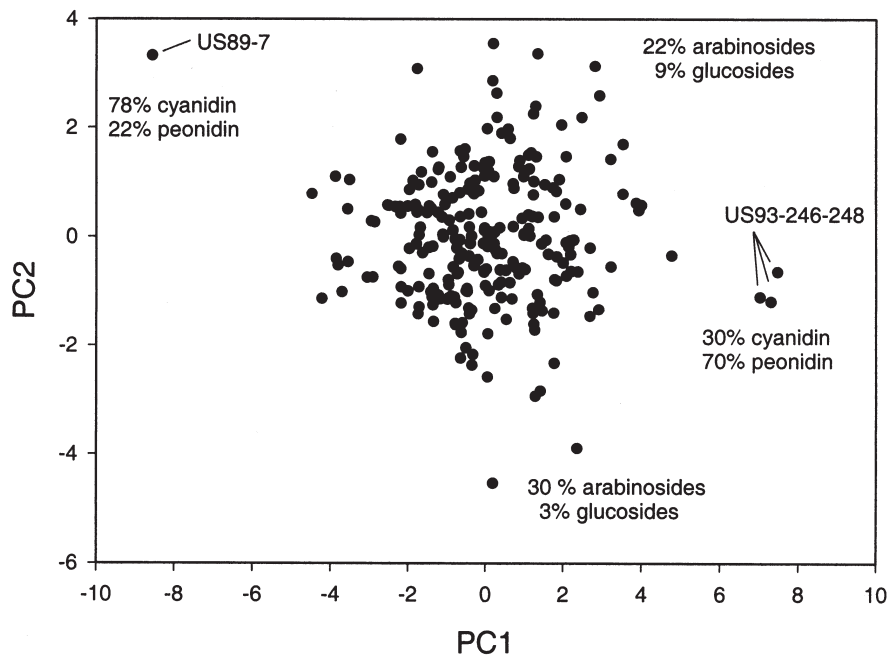

Fig. 1. Plot of the 1998 accession means (across two harvest dates) of first (PC1) and second principal (PC2) components derived from the levels of proportions of the six major cranberry anthocyanins.

of accessions were within the range of -4 and 4 for PC1, having cyanidin/peonidin proportions of $66 / 34$ (2:1) to $42 / 58$ (0.7:1). One apparent outlier was accession US89-7 which had $78.3 \%$ cyanidin ( $21.7 \%$ peonidin) anthocyanins or a 3.6:1 cyanidin:peonidin ratio. There was also another outlier group (US93-246, 247 \& 248) located at $\mathrm{PC} 1>7$, having a relatively low proportion (30\%) of cyanidin (70\% peonidin) anthocyanins, or a $0.5: 1$ cyanidin:peonidin ratio. Accessions US93-246, 247 and 248 were found to have identical randomly amplified polymorphic DNA (RAPD) and sequence characterized amplified regions (SCAR) fingerprints suggesting that they are, in fact, identical clones (Novy et al., 1994; Polashock, unpublished data). The ratio of proportions of arabinoside to glucoside anthocyanins ranged from 30.4:2.8 (US89-10) to 20.4:8.4 (US93-223) arabinose:glucose or ratios of 10:9 to 2.4:1, respectively. PC3 was significantly negatively correlated with the proportion of galactoside anthocyanins $(r=-0.74)$. PC3 values ranged from -1.9 to 1.6 , with galactoside anthocyanin proportions of 63.5 (US92-11) to 77.4 (US88-78) percent, respectively. Correlation of the principal component means (by accession) between the two years (78 genotypes) were highly positively significant, with $r=-0.96$ (negative due to sign reversal), 0.92 and 0.78 for $\mathrm{PC} 1,2$, and 3, respectively.

Peonidin anthocyanin proportions significantly increased with later harvest dates for both years, particularly with the peonidin arabinosides and galactosides (Fig. 2). Within harvest dates there was a positive correlation between peonidin-3-galactosides with total anthocyanins, while cyanidin-3-galactosides were negatively correlated with total anthocyanins (data not shown). Proportions of peonidin anthocyanins were significantly positively correlated with total anthocyanins for the September harvest dates for 1998 $(r=0.14, P<0.01)$ and $1999(r=0.32, P<0.01)$.

Clones (VARIETIES) COMMON TO BOTH BEDS. Clones or varieties were identified to be present in both germplasm beds through RAPD and SCAR fingerprinting technology (data not shown). Bed and variety effects were highly significant for PC1, 2 and 3 (Table 5). Harvest date effects were highly significant for PC1 and 3. Interaction effects among the main effects were not significant, including variety by harvest date. Bed 1 exhibited a higher PC1 value (1.5) than Bed $4(0.0)$ indicating a higher proportion of the peonidin anthocyanins. The later harvest date also exhibited higher

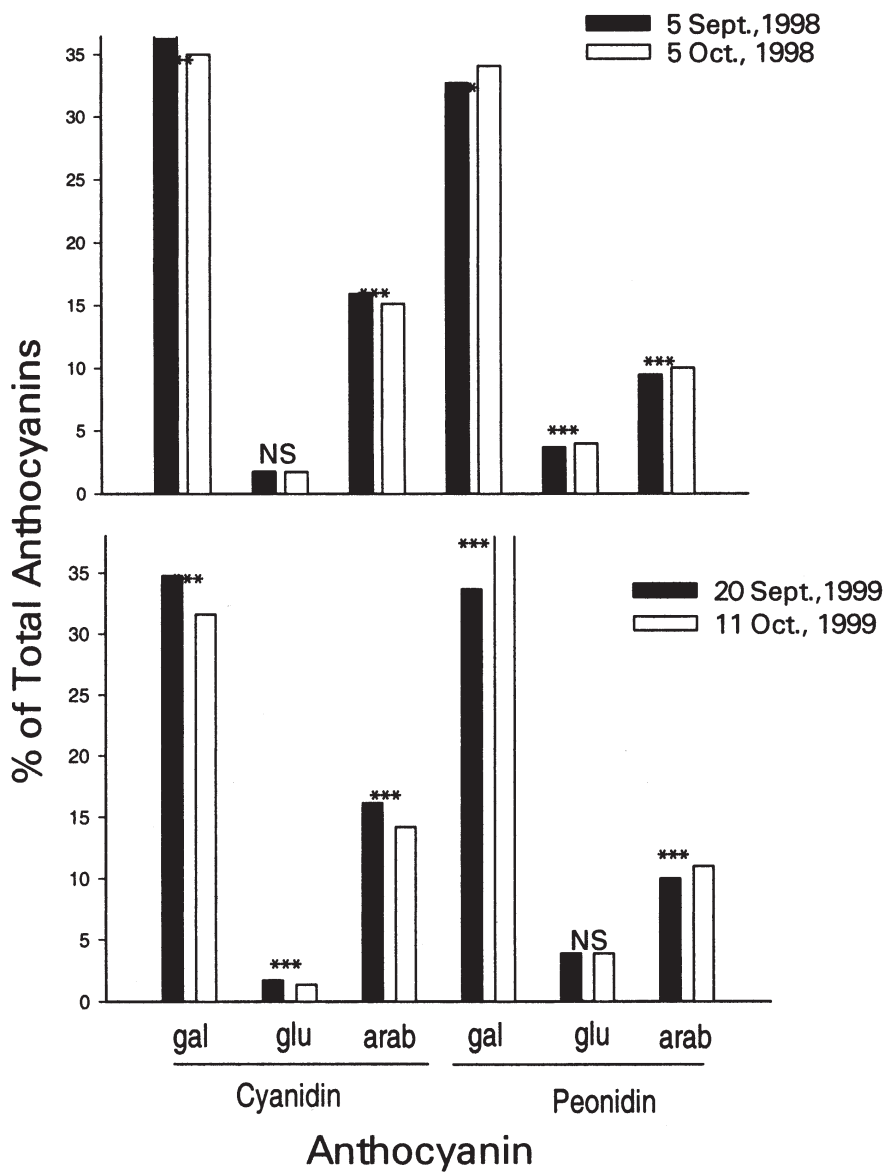

Fig. 2. Mean (across accessions) proportions of six cranberry anthocyanins for two harvest dates in 1998 and 1999.

proportions of peonidin anthocyanins. Both bed and variety effects were significant for PC2 suggesting both environmental and genetic effects are significant for arabinoside versus glucoside proportions. Bed, variety and harvest date effects were significant for PC3 indicating the proportions of galactosides versus arabinoside/glucoside have both environmental and genetic effects.

The ranges of proportions of the six anthocyanins of the common cultivars Stevens, Early Black, and Ben Lear are 30\% to $41 \%$ (cyanidin-3-galactoside), $1 \%$ to $2 \%$ (cyanidin-3-glucoside), $13 \%$ to $17 \%$ (cyanidin-3-arabinose), $29 \%$ to $40 \%$ (peonidin-3-galactoside), $3 \%$ to $5 \%$ (peonidin-3-glucoside) and $8 \%$ to $11 \%$ (peonidin-3-arabinoside) (Table 6). Cyanidin to peonidin anthocyanin ratios (cyd: pnd) varied from 0.8:1 ('Ben Lear') to 1.5:1 ('Early Black'). The

Table 5. ANOVA (Type III sums of squares) for principal components $\mathrm{PC} 1,2$, and 3 from principal component analysis of anthocyanin proportions from nine varieties as determined by SCAR fingerprint established in two cranberry beds.

\begin{tabular}{lcccc}
\hline & & \multicolumn{3}{c}{ Mean square } \\
\cline { 3 - 5 } Source & df & PC1 & PC2 & PC3 \\
\hline Bed & 1 & $14.9^{* * *}$ & $35.9^{* * *}$ & $4.0^{* * *}$ \\
Variety & 8 & $9.3^{* * *}$ & $5.7^{* * *}$ & $0.1^{* * *}$ \\
Bed $\times$ variety & 6 & 1.3 & 0.4 & 0 \\
Harvest date (HD) & 1 & $18.3^{* * *}$ & 0.7 & $38.7^{* * *}$ \\
Bed $\times$ HD & 1 & 1.5 & 0.3 & 0 \\
Variety $\times$ HD & 8 & 3.2 & 0.2 & 0 \\
Error & 32 & 1.3 & 0.3 & 0.01 \\
\hline
\end{tabular}

*** Significant at $P<0.001$. 
Table 6. Means (across 2 years) of proportions of the six anthocyanins and absolute total anthocyanins for selected cranberry germplasm accessions and cultivars.

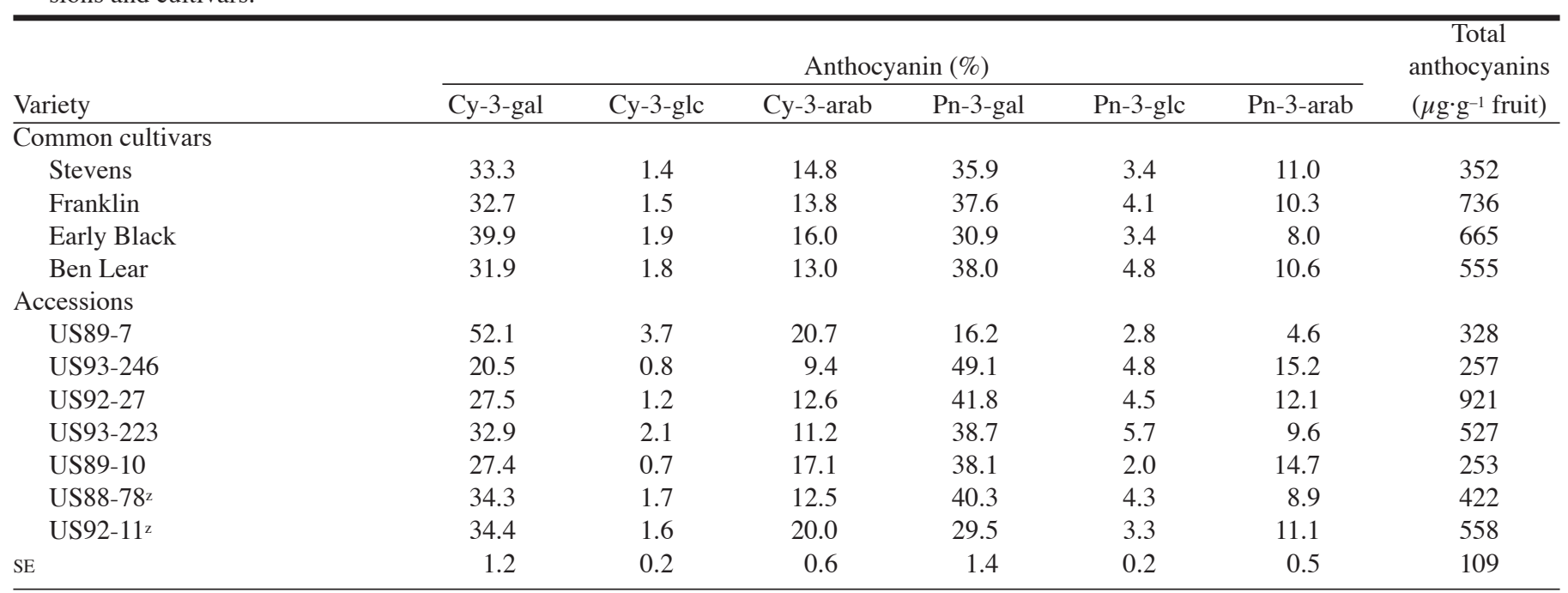

${ }^{\mathrm{z}}$ Data from 1998 only.

most widely planted cultivar Stevens had about equal proportions, 1:1 (1998) and 0.9:1 (1999), of cyanidin to peonidin anthocyanins (Table 6). Accession US89-7 had the greatest proportion of cyanidin anthocyanins of 78\% (1998), or a cyd:pnd ratio of 3.6:1. In 1999 the proportion of cyanidin anthocyanins was slightly lower at $75 \%$ (3: 1). Accession US93-246 (also accessions US93-247-248) exhibited the low range $(31 \%)$ of cyanidin anthocyanins or a ratio of 0.5 : 1, cyd:pnd. Accession US93-246, however, was relatively low in overall anthocyanin content (Table 6). In contrast, the accession having the next lowest cyanidin proportion and relatively high anthocyanin production was US92-27, which had $\approx 43 \%$ cyanidin anthocyanins (Table 6).

Proportions of arabinoside anthocyanins of the major cultivars range was narrow ranging from $24 \%$ to $26 \%$. The range of arabinoside proportion across accessions was $\approx 21 \%$ (US93-223) to $32 \%$ (US89-10) (Table 6). Galactoside proportions of the major cultivars ranged narrowly between $69 \%$ to $71 \%$. The highest proportion of galactoside anthocyanins was $\approx 75 \%$ (US88-78), and lowest was $\approx 64 \%$ (US92-11) (Table 6).

\section{Discussion}

Hong and Wrolstad (1986) reported cyanidin anthocyanin proportions ranged from $53 \%$ (1.1:1, cyanidin:peonidin)for the cultivar Howes to 65\% (1.8:1, cyanidin:peonidin) for 'Early Black'. Sapers and Hargrave (1987) reported cyanidin-3-galactoside percentages ranging from $18.4 \%$ (cultivar not given) to $33.7 \%$ ('Early Black'). The 'Early Black' accession in this study exhibited a higher percentage (39\% to $41 \%$ ) of cyanidin-3-galactoside than Sapers and Hargrave (1987). Genetic identity of cranberry varieties, however, is problematic (Novy et al., 1994; Novy and Vorsa, 1995), and it is not certain whether the 'Early Black' clones between these two studies are in fact identical genotypes. In addition, although profiles were similar across the two years of this study, differences in growing year environments are also possible. The proportions of cyanindin-3-galactoside, cyanindin-3-arabinoside, peonidin-3galactoside, and peonidin-3-arabinoside for the cultivar Franklin, however, appear to be similar between the studies with the exception that peonidin anthocyanin proportions appear to be slightly higher in Sapers and Hargrave (1987). This study of a broader array of germplasm identified a broader range of variation of anthocyanin profiles in cranberry.

The anthocyanin biochemical pathway, which shares early steps with the flavonol and proanthocyanidin pathways, is a complex pathway consisting of numerous structural genes (Holton and Cornish, 1995). While enzymes early in the flavonoid pathway, (prior to chalcone isomerase), are thought to be encoded by large multigene families, it has been suggested that enzymes after the chalcone synthase step are encoded by single active genes (Sparvoli et al., 1994). However, genetic evidence suggests this may not be the case (Jonsson et al., 1983). Dihydroflavonol-4-reductase in cranberry is encoded by a least two loci (Polashock et al., 2002). In maize, a number of regulatory genes controlling expression of the anthocyanin pathway structural genes have been identified (Dooner and Robbins, 1991). Boss et al. (1996) found almost all the flavonoid biosynthetic genes were expressed in many plant tissues in grape, whereas, glucose-flavonoid-3-O-glucosyltransferase, the first step from anthocyanidin to anthocyanin production, was only expressed in the fruit skin. A mutation in a regulatory gene controlling the expression of a number of anthocyanin pathway genes including UDP-glucose:flavonoid 3- $O$-glucotransferase was found in red-sports of white grapes (Kobayashi et al., 2001).

The large variation (150-fold) in overall anthocyanin content in cranberry fruit suggests that considerable genetic variation is present for overall expression of the anthocyanin pathway. However, variation in anthocyanin content has to be viewed in the context of all the factors that can contribute to variation in whole fruit samples. Anthocyanins are located predominately in the fruit skin in cranberry, appearing to be located in the outermost layer of elongated epidermal cells and the inner layer of larger cells (Sapers et al., 1983a). Thus, variation in overall anthocyanin content of whole fruit samples is at least partially a function of fruit size, i.e. amount of surface area, which is also a function of variety (Vorsa and Welker, 1985). Recognized environmental effects which enhance anthocyanin expression include water, light and temperature stresses and nutrition. Furthermore, there is a negative correlation between yield and many of the flavonoid compounds, including anthocyanins, proanthocyanidins, and total phenolics (Vorsa et al., 2000). Environmental stresses reduce fruit size, and thus yield. Narrow sense heritability for total anthocyanins has been estimated as high as $h^{2}=0.72$ from parent-offspring regression suggesting additive genetic effects contribute to major differences in overall 
anthocyanin production (Vorsa, unpublished data).

Analysis of the cranberry germplasm anthocyanin profiles offers insight into the genetic and phenological effects that play a role in the partitioning of flavonoid resources. Contrary to the conclusions of Sapers and Hargrave (1987), this study suggests there is significant genotypic variation for individual anthocyanin proportions. Evidence for both qualitative and quantitative variation for proportions of the aglycone (in the conjugated form), cyanidin versus peonidin, was found. Accession US89-7 producing $\approx 80 \%$ cyanidin anthocyanins is an outlier (Fig. 1) suggesting possible homozygosity for a defective structural methyltransferase gene or a regulatory gene of methyltransferase. Jonsson etal.(1983) suggested at least two anthocyanin methyltransferases were present in Petunia hybrida under the control of four regulatory genes. If more than one methyltransferase locus exists in cranberry, then the simplest explanation would be that a regulatory gene is responsible for the relatively low peonidin content in US89-7. Significant quantitative variation is also present, suggesting more subtle allelic differences for structural and/or regulatory genes.

Grisebach (1982) suggests that methylation occurs at the cinnamic acid stage or later at the chalcone-flavonone stage. The greater proportions of peonidin in later harvests and positive correlation with overall anthocyanin content indicates that the methylation step in cranberry occurs post-glycosylation by methyltransferases as suggested by Holton and Cornish (1995). Do et al. (1995) found the Vitis vinifera 3-O-glucosyltransferase to have the highest activity with cyanidin, and considerably reduced activity with both 3 ' and 5' methylated anthocyanidins, peonidin and malvidin. The positive correlation of peonidin anthocyanin proportion with total anthocyanins and later harvest dates suggests that the expression of methyltransferase(s) is not or only partially coupled with expression of earlier pathway genes. If methylation occurs early in the pathway, i.e., at chalcone isomerase or synthase steps, then one would not expect to have phenological effects influencing proportions of cyanindin versus peonidin proportions.

Apparent genetic variation was found for glycosylation profiles. The proportions of arabinosides versus glucosides (PC2) accounted for a major portion of the variation in glycosylation, followed by arabinosides/glucosides versus galactosides (PC3). In many species, anthocyanidins are conjugated to glucose by UDP glucose:flavonoid 3-O-glucosyltransferase to form anthocyanidin 3-glucosides followed by further modification to other glycosides (Holton and Cornish, 1995). Glycosyltransferases represent a large supergene family and appear to have fairly broad affinity for substrates, but exert regioselectivity and regiospecificity for the sugar acceptor (Vogt and Jones, 2000). Multiple glucosyltransferases have been identified in Petunia hybrida with specificity for both, the substrate and sugar donor, as well as differential expression in various tissues. An UDP-glucose:flavonoid 3- $O$-glucosyltransferase isolated from Vitis vinifera did not exhibit activity with UDP-galactose (Ford et al., 1998). Similarly, evidence suggests that in some species such as Eustoma grandiflorum, which produces predominantly 3-Ogalactosylated flavonols, that 3-O-glucosylated flavonols can be increased substantially with transformation and expression of a UDP-glucose:flavonoid-3-O-glucosyltransferase (Schwinn et al., 1997). It has been suggested that the supply and quality of nucleotides would be conserved during evolution leading to high substrate specificity for the sugar donor (Vogt and Jones, 2000). Thus, it could be postulated that cranberry has up to three glycosyltransferase enzymes including galactosyltransferase, arabinosyltransferase and glucosyltransferase, the genes for which would be located at respective loci. Alternatively, one glycosyltransferase encoded by one locus may conjugate all three sugars, with allelic variation yielding enzymes having different efficiencies $(\mathrm{Kms})$ for the various sugars. Both genotypic and environmental (bed) effects impact (PC2 and PC3) glycosylation profiles. Phenological effects were only significant for galactoside versus glucoside/arabinoside trade-offs (PC3), suggesting that galactosyltransferase expression may not be coupled with glucosylation/arabinosylation. Since glucose and arabinose are six and five carbon sugars, respectively, with identical stereochemistry for the five hydroxyl groups, it could be reasoned that both sugars are conjugated by one glycosyltransferase enzyme in cranberry. The significant trade-offs between glucosides versus arabinosides (PC2) may reflect allelic differences in nucleotide affinities or carbohydrate availability.

In conclusion, the significant genetic variation for cranberry anthocyanin profiles provides opportunities to breed for specific anthocyanin profiles. The greatest opportunity appears to be for proportion of cyanidin versus peonidin anthocyanins, with particularly high $(80 \%)$ cyanidin profiles the most readily achievable since it may be a qualitative trait. Cranberry varieties with not only high overall anthocyanin levels, but with increased proportions of cyanidin anthocyanins would be expected to have higher antioxidant capacity potential due to the apparently higher antioxidant capacity of cyanidin relative to peonidin that was reported by Wang et al. (1997). Although apparent genetic variation exists for glycosylation profiles, the variation in the current germplasm collection may not be practically significant.

Since cranberry is an asexually propagated crop, the lack of numerous sexual generations, reduces the opportunity of exposing mutations as homozygous recessive traits. Screening undomesticated germplasm may provide additional unique profiles and genes for breeding. However, opportunities should exist with inbreeding for identifying variant regulatory or structural alleles for glycosylation and other flavonoid pathway genes.

\section{Literature Cited}

Boss, P.K., C. Davies, and S.P. Robinson. 1996. Expression of anthocyanin biosynthesis pathway genes in red and white grapes. Plant Mol. Biol. 32:565-569.

Cao, G., E. Sofic, and R.L. Prior. 1996. Antioxidant and pro-oxidant behavior of flavonoids: Structure-activity relationships. Free Radical Biol. Med. 22:749-760.

Do, C.B., F. Cormier, and Y. Nicolas. 1995. Isolation and characterization of a UDP-glucose:cyanidin 3-O-glucosyltransferase from grape cell suspension cultures (Vitis vinifera L.). Plant Sci. 112:43-51.

Dooner, H.K. and T.P. Robbins. 1991. Genetic and developmental control of anthocyanin biosynthesis. Annu. Rev. Genet. 25:173-199.

Ford, C.M., P.K. Boss, and P. B. Hoj. 1998. Cloning and characterization of Vitis vinifera UDP-glucose: Flavonoid 3-O-glucosyltransferase, a homologue of the enzyme encoded by the maize bronze- 1 locus that may primarily serve to glucosylate anthocyanindins in vivo. J. Biol. Chem. 273:9224-9233.

Gee, J.M. M.S. DuPont, A.J. Day, G.W. Plumb, G. Williamson, and I.T. Johnson. 2000. Uptake of quercetin glycosides involves deglycosylation and interaction with sodium-dependent glucose transport pathway in rat small intestine. J. Nutr. 130:2765-2771.

Grisebach, H. 1982. Biosynthesis of anthocyanins, p. 69-92. In: P. Markakis (ed.). Anthocyanins as food colors. Academic Press, New York.

Hollman., P.C.H. and M.B. Katan. 1997. Absorption, metabolism, and health effects of dietary flavanoids in man. Biomedicine Pharmacotherapy 51:305-310.

Hollman, P.C.H., J.M.P. van Trijp, M.N.C.P. Buysman, M.S. v.d. Gaag, M.J.B. Memgelers, J.H.M. de Vries, and M.B. Katan. 1997. Relative bioavailability of the antioxidant flavonoid quercetin from various foods. FEBS Lett. 418:152-156. 
Holton, T.A. and E.C. Cornish. 1995. Genetics and biochemistry of anthocyanin biosynthesis. Plant Cell 7:1071-1083.

Hong, V. and R.E. Wrolstad. 1986. Cranberry juice composition. J. Assn. Off. Anal. Chem. 69:199-207.

Hong, V. and R.E. Wrolstad. 1990. Use of HPLC separation/photodiode array detection for characterization of anthocyanins. J. Agr. Food Chem. 38:708-715.

Jonsson, L.M.V., P. de Vlaming, H. Wiering, M.E.G. Aarsmanard and A.W. Schram. 1983. Genetic control of anthocyanin-O-methyltransferase activity in flowers of Petunia hybrida. Theor. Appl. Genet. 66: 349-355.

Kobayashi, S., M. Ishimaru, C.K. Ding, H. Yakushiji, and N. Goto. 2001. Comparison of UDP-glucose: Flavonoid3-O-glucosyltransferase (UFGT) gene sequences between white grapes (Vitis vinifera) and their sports with red skin. Plant Sci. 160:543-550.

Lamuela-Raventos, R.M. and A.L. Waterhouse, 1994. A direct HPLC separation of wine phenolics. Amer. J. Enol. Viticult. 45:1-5.

Mizuma, T., K. Ohta, and S. Awazu. 1994. The $\beta$-anomeric and glucose preferences of glucose transport carrier for intestinal active aborption of monosaccharide conjugates. Biochim. Biophys. Acta 1200:117-122.

Novy, R.G., C. Kobak, J. Goffreda, and N. Vorsa. 1994. RAPDs identify varietal misclassification and regional divergence in cranberry ( $\mathrm{Vac}$ cinium macrocarpon Ait.). Theor. Appl. Genet. 88:1004-1010.

Novy, R.G. and N. Vorsa. 1995. Identification of intracultivar genetic heterogeneity in cranberry using silver-stained RAPDs. HortScience 30:600-604.

Polashock, J.M., R.J. Griesbach, R.F. Sullivan, and N. Vorsa. 2002. Cloning of a cDNA encoding the cranberry dihydroflavonol-4-reductase (DFR) and expression in transgenic tobacco. Plant Sci. 163: 241-251.

Sapers, G.M. and D.L. Hargrave. 1987. Proportions of individual anthocyanins in fruits of cranberry cultivars. J. Amer. Soc. Hort. Sci. 112:100-104.

Sapers, G.M., S.B. Jones, and G.T. Maher. 1983a. Factors affecting the recovery of juice and anthocyanin from cranberies. J. Amer. Soc.
Hort. Sci. 108:246-249.

Sapers, G.M., J.G. Phillips, H.M. Rudolf, and A.M. DiVito. 1983b. Cranberry quality: Selection procedures for breeding programs. J. Amer. Soc. Hort. Sci. 108:241-246.

Satue-Gracia, M.T., M. Haeinonen, and E.N. Frankel. 1997. Anthocyanins as antioxidants on human low-density lipoprotein and lecithin-liposome systems. J. Agr. Food Chem. 45:3362-3367.

Schwinn, K.E., K.M. Davies, S.C. Deroles, K.R. Markham, R.M. Miller, J.M. Bradley, D.G. Manson, and N.K. Given. 1997. Expression of an Antirrhinum majus UDP-glucose: Flavonoid-3-O-glucosytransferase transgene alters flavonoid glycosylation and acylation in lisianthus (Eustoma grandiflorum Grise.). Plant Sci. 125:53-61.

Setchell, K.D.R., N.M. Brown, P. Desai, L. Zimmer-Nechemias, B.E. Wolfe, W.T. Brashear,A.S. Kirschner, A. Cassidy, and J.E. Heubi. 2001. Bioavailability of pure isoflavones in healthy humans and analysis of commercial soy isoflavone supplements. J. Nutr. 131:1362S-1375S.

Sparvoli, F.C., C. Martin, A. Scienza, G. Gavazzi and C. Tonelli. 1994. Cloning and molecular analysis of structural genes involved in flavonoid and stilbene biosynthesis in grape (Vitis vinifera) Plant Mol. Biol. 24:743-755.

Vogt, T. and P. Jones. 2000. Glycosyltransferases in plant natural product synthesis:characterizarion of a supergene family. Trends Plant Sci. 5: 380-386.

Vorsa, N. and W.V. Welker. 1985. Relationship between fruit size and extractable anthocyanin content in cranberry. HortScience 20: 402-403.

Vorsa, N., J. Polashock, A. Howell, D. Cunningham, and R. Roderick. 2000. Evaluation of fruit chemistry in cranberry germplasm: Potential for breeding varieties with enhanced health constituents. 7 th Intl. Symp. Vaccinium Culture, termas de Chillán, Chillán, Chile, 4-9 Dec.

Wang, H. G. Cao, R.L. Prior. 1997. Oxygen radical absorbing capacity of anthocyanins. J. Agr. Food Chem. 45:304-309.

Wrolstad, R.E., J.D. Wightman, and R.W. Durst. 1994. Glycosidase activity of enzyme preparations used in fruit juice processing. Food Technol. (Nov.):90, 92-94, 96, 98. 